\title{
Phantastik und Philosophie in Guillermo del Toros El laberinto del fauno
}

Cornelia RuHe

La guerre s'est assoupie, un oeil toujours ouvert.

(NUIT ET BROUILLARD [1955])

\section{ZWISCHEN GrossprojeKtEN UND PERSÖNLICHEN ARBEITEN}

Guillermo del Toro ist ein Multitalent. Als Regisseur, Produzent, Drehbuchund seit kurzem auch Romanautor ${ }^{1}$ feiert er international Erfolge. Mit seinem ersten Film La invención de Cronos (1993) ist er zunächst vor allem Fans von Genrefilmen ein Begriff. Er schafft allerdings bereits mit dem nächsten Film den Sprung nach Hollywood, wo er mit internationalen Stars Filme mit großem Budget dreht (Mimic [1997], Blade II [2002], Hellboy [2004], Hellboy - The Golden Army [2008]). Sie gehören den Genres des Horror- oder Actionfilms an, darunter sind aber auch Comic-Verfilmungen. Die Kritiker heben stets hervor, dass sich diese Filme durch eine originelle Handschrift auszeichnen. ${ }^{2}$ Del Toro selbst betont gerne, dass er diese Groß-

1 Der Roman von Guillermo del Toro und Chuck Hogan mit dem Titel Nocturna bzw. The Strain wurde im Juni 2009 gleichzeitig auf Englisch und auf Spanisch publiziert, ebenso der zweite Teil, Oscura/The Fall, der im September 2010 erschien. Der dritte Teil der Trilogie, Eterna/The Night Eternal, soll im Oktober 2011 veröffentlicht werden.

2 So z.B. Michael Althen: „Den Fans des Horror- und Fantasy-Genres ist del Toro schon seit längerem bekannt als Mann, der im Einerlei von Blut und Gewalt immer wieder ungewöhnliche Poesie und enorm nachhaltige Bilder entdeckt.“ (Althen 2007: 35) 
projekte für Hollywood-Studios gemacht habe, um Geld für kleinere, europäische Filme zu sammeln, die ihm persönlich am Herzen liegen. ${ }^{3}$

Der zweite dieser persönlichen Filme, El laberinto del fauno, ${ }^{4}$ oder, um den homonymen deutschen und internationalen Titel zu nennen, Pans Labyrinth (2006), hatte zwar ein relativ bescheidenes Budget von nur 19 Millionen US-Dollar, entwickelte sich aber zum kommerziell erfolgreichsten spanischsprachigen Film aller Zeiten mit einem Einspielergebnis von 83 Millionen US-Dollar. Der Film lief im Wettbewerb der Filmfestspiele von Cannes, wo der Regisseur und seine Crew mit 22 Minuten stehender Ovationen gefeiert wurden (vgl. Wikipedia), gewann drei Oscars und zahlreiche weitere Preise, ${ }^{5}$ ein Ausweis der Anerkennung auch durch die Kritiker.

Pans Labyrinth verarbeitet Elemente aus Phantastik, Mythologie und aus dem del Toro vertrauten Genre des Horrorfilms und thematisiert nicht zuletzt Aspekte der jüngeren Geschichte Spaniens. Die Handlung spielt 1944 im spanischen Hinterland, wo eine Truppe von Partisanen ungeachtet des seit 1939 offiziell beendeten Krieges weiterhin versucht, sich den siegreichen Franquisten zu widersetzen. Am Ende des Films steht der Sieg der Partisanen über Kapitän Vidal und seine Truppen.

\section{2 ,AN ANTI-FASCIST FAIRY TALE SET IN 1944“6}

Für den aufmerksamen Zuschauer verweist bereits der Titel des Films explizit nicht nur auf einen möglichen magisch-phantastischen Kontext, sondern auch auf den Spanischen Bürgerkrieg. Max Aubs Romanzyklus über die Guerra Civil (1943-1967) trug den Titel El laberinto mágico. Die Metapher

3 „Since the early 1990s, del Toro has divided his film-making between personal European projects (the modern vampiric chiller Cronos in 1993; the ghostly Spanish Civil War fable The Devil's Backbone in 2001) and big-budget Hollywood hits (ongoing comic-book franchises Blade II in 2002, and Hellboy in 2004).“ (Kermode 2006)

4 Der erste persönliche Film ist El espinazo del diablo (2001), der ebenso wie $E l$ laberinto del fauno zur Zeit des spanischen Bürgerkriegs bzw. unmittelbar danach spielt. Beide Filme werden von del Toro als Teil einer noch nicht abgeschlossenen Trilogie betrachtet.

5 Darunter sieben Goyas (Beste Nachwuchsdarstellerin, Bestes Originaldrehbuch, Beste Kamera, Bester Schnitt, Beste Maske, Bester Ton und Beste Spezialeffekte), neun Ariels (Bester Film, Beste Regie, Beste Schauspielerin, Beste Fotografie, Beste künstlerische Leitung, Bestes Kostüm, Bestes Make-up, Beste Effeke, Beste Originalmusik) und drei Preise der British Academy of Film and Television Arts (BAFTA; für den besten nicht englischsprachigen Film, für Kostüm und für Make-up). Die Oscars erhielt der Film für Beste Kamera, Bestes Szenenbild und Bestes Make-up.

6 Interview mit Guillermo del Toro in $U G O$ Giversitgätsbibliothek Mannheim 
wurde von Gerald Brenan aufgenommen, der seinen bekannten historischen Text über den Spanischen Bürgerkrieg Spanish Labyrinth (1943) nennt.

Noch vor dem ersten Bild ertönt ein offensichtlich erschwertes Atemgeräusch. Man sieht sodann das sterbende Mädchen Ofelia, deren Atem der Zuschauer vorher bereits gehört hat, am Rande des titelgebenden Labyrinths liegen. Ihr Blut tropft allerdings nicht nach unten, sondern fließt zu ihr zurück - die Zeit wird zurückgedreht, damit der Film beginnen kann. Einerseits könnte man hier ein konventionelles Signal für die nun erfolgende Rückblende sehen, die den ganzen Film über andauert und die in dem für die Partisanen optimistischen Ende des Films gipfelt, das zum ambivalenten Charakter des Films passt. Im Gegensatz zu anderen filmischen Darstellungen, die den historischen Kampf der Partisanen ins Zentrum rücken, überleben sie hier nicht nur, sondern schlagen sogar die gegnerischen Truppen vernichtend. Ihr Sieg hat allerdings einen hohen Preis: Ofelia muss sterben, damit er möglich wird. Das rückwärts fließende Blut dreht folglich nicht nur für Ofelia die Zeit zurück, sondern ermöglicht es auch den eigentlich bereits geschlagenen Partisanen, ihren Kampf noch einmal mit neuer Hoffnung aufzunehmen.

Andererseits, so die hier vertretene These, handelt es sich nicht allein um eine Rückblende; vielmehr ist der Film von seinem Anfang wie von seinem Ende her um ein Zentrum organisiert, das um die Themen Wahrnehmung und Kontrolle kreist.

Die Protagonistin des Films, Ofelia (Ivana Baquero), befindet sich mit ihren elf Jahren in der Übergangsphase von der Kindheit zur Jugend. Transitorische Zustände sind darüber hinaus für den Film auf verschiedenen Ebenen kennzeichnend: ${ }^{7}$ Die historische Situation ist eine des Übergangs von der Kriegs- zur Nachkriegszeit, in der noch Kämpfe zwischen Partisanen und Franquisten ausgetragen werden; Carmen (Ariadna Gil), Ofelias Mutter, ist hochschwanger und befindet sich damit physisch in einem Übergangsstadium, das in ihrem Fall die ganze Spannbreite von Geburt und Tod umfassen wird. Zentral für den Film ist insbesondere die Transition zwischen realistischer, historisch klar situierter Welt und der phantastischen Ebene, die hier parallel zueinander etabliert werden und ständig ineinander fließen.

Ofelia ist die einzige Person, der es möglich ist, zwischen beiden Welten hin- und herzuwechseln. Sie kann umso besser als Mittlerin zwischen den Welten agieren, als sie sich selbst noch in einem Übergangsstadium befindet. Obwohl sie bereits elf Jahre alt ist, hat sie, so scheint es, das magische

7 Das Labyrinth selbst ist für del Toro ein Symbol für transitorische Zustände: „Then, as time goes by, I think the labyrinth becomes a symbol of transition, of a trip. In many cases during the Holy Wars, the labyrinth becomes a transit that is supposed to allow you to walk along a path while you are meditating or praying. Inevitably, you will end up in the center. [...] As Borges, Greek mythology, and oral folklore will tell you, the labyrinth reflects the values of its time." (In Fuchs 2007) 
Alter noch nicht gänzlich hinter sich gelassen. Dieser scheinbare Entwicklungsrückstand wird immer wieder von ihrer Mutter, aber auch von ihrem Stiefvater, dem Hauptmann Vidal (Sergi López), moniert. Beide sehen den Grund für dieses Defizit in Ofelias übermäßiger Lektüre von Märchen.

In den ersten Szenen des Films wird dieser Aspekt der kindlichen Protagonistin offensichtlich: Eine Stimme aus dem Off erzählt eine Geschichte von einer Prinzessin namens Moana aus dem „reino subterraneo“, die von der Welt der Menschen träumte, der der Aufstieg ans Tageslicht gelang und die dort, geblendet vom Licht, ihre Herkunft vergaß und daher unfähig zur Rückkehr war. Es gelte nun, diese Prinzessin wiederzufinden und in die ,Unterwelt‘ zurückzubringen. Die Geschichte bricht ab, die nächste Einstellung zeigt Ofelia, die in einem Märchenbuch blättert, sehr zum Unwillen ihrer Mutter, die sie dafür tadelt (,,Ya eres muy mayor para llenarte la cabeza con tantas zarandajas" [00:03:03]).

Aus dem Zusammenspiel der Off-Stimme mit dem Bild des lesenden Mädchens könnte man schließen, dass die vorgetragene Geschichte Ofelias Buch entstamme. Sie selbst habe es soeben gelesen, passe im Folgenden ihre Welt der des Märchens an und träume sich selbst in die Rolle der verschollenen Prinzessin Moana. Die Geschichte entstammt aber nicht dem Text des Märchenbuchs, das somit zum Auslöser für die Handlung wird, sondern seiner Illustration - also dem zum Film passenden Bildmedium. Diesem Bild in ihrem Buch - einem von vier Feen umschwärmten Mädchen - wird Ofelia sich im Verlauf des Films anverwandeln.

\section{Doppelte Prüfungen}

Das Thema der Wahrnehmung und das der Kontrolle werden gleich zu Beginn des Films in einer Szene eingeführt, die sich metapoetisch als Reflexion über die Arbeit des Interpreten lesen lässt: Die Autofahrt von Ofelia und ihrer Mutter zur Mühle, in der Hauptmann Vidal, ihr zweiter Ehemann, sie erwartet, wird unterbrochen, da die hochschwangere Carmen sich übergeben muss. Ofelia steigt aus und findet auf der Straße einen eigenartigen Stein, der so bearbeitet ist, dass er wie ein Auge aussieht. Als sie den Weg verlässt und einige Schritte in den Wald hineinläuft, entdeckt sie eine moosüberwachsene, steinerne Statue mit einem riesigen, wie zum Schrei geöffneten Mund. Der Statue, die der Zuschauer retrospektiv als eine Darstellung der für den Film zentralen Figur des Fauns erkennen wird, fehlt ein Auge. Ofelia setzt den Stein, den sie gefunden hat, an der entsprechenden Stelle ein, vervollständigt so die Statue und befähigt sie wieder zur vollständigen Wahrnehmung und zur Kontrolle ihrer Umwelt. Als habe sie damit einen Mechanismus betätigt, entschlüpft dem Mund der Statue nun eine große Stabheuschrecke, die als Ofelias Führerin in die Geschichte dienen wird. Ofelia meint bereits jetzt, darin eine Fee zu erkennen, was sie auch ihrer 
Mutter mitteilt, die daran allerdings keinerlei Interesse zeigt, sondern nur Augen für Ofelias schmutzig gewordene Schuhe hat.

Aus einer metapoetischen Perspektive liest sich die Passage als ein Hinweis auf die Arbeit des Interpreten des Films - ein Element wird gefunden, an der richtigen Stelle eingesetzt und bringt so die Handlung wie auch die weitere Interpretation in Gang. Die Voraussetzung dafür ist aber, dass man, anders als die pragmatische Mutter, zur Wahrnehmung der gleichsam poetischen Implikationen des Alltags befähigt ist und sich den Blick darauf nicht durch prosaische Details wie schmutzige Schuhe verstellen lässt.

Das Auge, das Ofelia der Statue einsetzt, um ihr so neues Leben bzw. neue Wahrnehmung einzuhauchen, korrespondiert mit einem anderen Auge, das sich am Ende des Films für immer schließen wird: Ofelias Stiefvater, der faschistische Hauptmann Vidal, dessen Wahrnehmung in scharfem Kontrast zu ihrer eigenen steht, wird am Ende des Films von den Partisanen gestellt und erschossen. Der Schuss trifft ihn dabei unterhalb des Auges, das in der Folge bricht. Dieses, blinde“ Auge Vidals spiegelt das fehlende Auge der Statue. Ebenso wie Ofelia am Anfang durch den Fund des Augen-Steins die Handlung in Gang setzt, so ist sie es auch, die sie zu Ende führt, indem sie am Ende den Tod ihres Stiefvaters mit verantwortet.

Ofelia sieht in der Stabheuschrecke, die sie später zu dem Faun führen wird, bereits bei ihrer ersten Begegnung eine Fee. Als die Heuschrecke in der ersten Nacht in der alten Mühle in ihrem Zimmer erscheint, zeigt Ofelia ihr in ihrem Märchenbuch ein Bild von einer Fee, und das Insekt gleicht sich vor ihren Augen diesem Bild an. Die Wirklichkeit ist mit den Augen Ofelias gesehen wandelbar, Dinge wie Lebewesen können mehr als eine Gestalt haben, können poetischen Charakter annehmen.

Zuvor, bei Ofelias und Carmens Ankunft in der alten Mühle, macht Ofelia zum ersten Mal Bekanntschaft mit ihrem Stiefvater. Der Film charakterisiert Vidal als einen Technokraten und durch seine Uniform als Faschisten, der selbst gefangen ist in dem von ihm vertretenen System und nicht zulassen kann, dass in seiner Umgebung dagegen verstoßen wird. Sein Fetisch ist die Uhr. Während Ofelia noch vor ihrem ersten Erscheinen im Film durch ihr schweres Atmen präsent war, wird Vidal durch ein weniger natürliches Geräusch charakterisiert: Der Zuschauer hört auf der Tonspur ein überlautes Ticken, noch bevor er die dazugehörige Uhr in der Hand Vidals sieht. In Erwartung des Eintreffens von Ofelia und Carmen blickt Vidal auf die Uhr und konstatiert ungehalten 15 Minuten Verspätung des Konvois. Die Kontrolle über die Zeit, die er mittels der Uhr zu haben meint, spiegelt Vidals manischen Kontrollwahn auf allen anderen Ebenen. Er ist stets darauf bedacht, sich jeden und alles untertan zu machen.

Der Rückzugsort des Hauptmanns in der Mühle ist konsequenterweise das Räderwerk. Mehrmals wird gezeigt, wie er sich in diesem Zimmer rasiert, während im Hintergrund das bedrohliche und übermächtige Mahlwerk mit seinen Zahnrädern zu sehen ist (Abb. 1). Er wird damit als Teil des Räderwerks des Faschismus eingeführt. 
In einer für die Charakterisierung der Figur wichtigen Szene wird Vidal gezeigt, wie er in diesem Raum, der in mehrfacher Hinsicht das Zentrum der Mühle ist, mit den geisterhaften Schatten der Mühlenräder im Hintergrund, seine Taschenuhr reinigt. Er hat dabei eine Uhrmacherlupe ins Auge geklemmt und betrachtet ehrfürchtig die Zahnräder der Uhr, bevor er den Deckel wieder schließt. Vidals nahezu fetischistische Fixierung auf Räderwerke und Uhren wird so auf der Bildebene mehrfach überdeutlich markiert.

\section{Abbildung 1: Vidal rasiert sich, im Hinter- grund das Mahlwerk der Mühle}

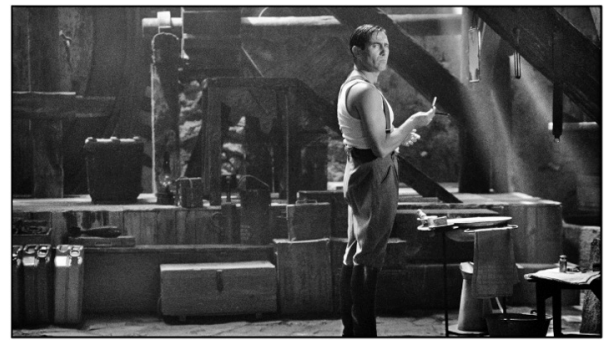

Quelle: @ Senator Film Verleih GmbH 2007

Bei einem Bankett Vidals mit den Honoratioren des Dorfes erzählt ein Gast, er habe Vidals Vater gekannt, der ebenfalls Offizier gewesen sei. Als er sich in einer ausweglosen Lage befunden habe, habe er, den Tod vor Augen, seine Taschenuhr zerschmettert, ,para que su hijo supiera como muere un valiente“ (00:40:52). Vidal bestreitet verärgert diese Geschichte. Am Ende des Films wird er dennoch diese von ihm geleugnete Geste seines Vaters zu wiederholen versuchen. Er, der mit dem beständigen Blick auf die Uhr lebt, verlangt von den Partisanen, die ihn töten werden, dass sie seinem eigenen Sohn später mitteilen mögen, wann und wie er gestorben sei. Seine Bitte wird ihm von der mit den Partisanen verbündeten Dienerin Mercedes verweigert: „Ni siquiera sabrá tu nombre.“(01:43:32) Die genealogische Linie, die Vidal über drei Generationen zu bewahren sucht, ja die er als Tradition etablieren möchte, wird unterbrochen. Bereits in diesem Bruch deutet sich an, dass der Film eine Loslösung von der spanischen Tradition, die in den Krieg geführt hat, sucht, hin zu einem Neuanfang.

Del Toros filmisches Universum weist eine Reihe von Konstanten auf, die seinen Filmen einen hohen Wiedererkennungswert verleihen. Der Regisseur ist fasziniert von mechanischen Apparaturen, ${ }^{8}$ allen voran Zahnrädern

8 Siehe dazu del Toros Aussagen in einem Interview: „Frage: Ihre Vorliebe für Mechanik zieht sich durch all ihre Filme hindurch, hier gibt es am Ende beim Auftauchen der Goldenen Armee wieder das von Ihnen so geschätzte Räderwerk zu sehen. Sie sammeln ja auch mechanisches Spielzeug. - Antwort del Toro: [...] Maschinen faszinieren mich, als Kind war das vor allem das Interesse an Auto- 
und Uhren, ebenso von Labyrinthen. ${ }^{9}$ Es ist aber nicht nur der Vorliebe des Regisseurs zu verdanken, dass El laberinto del fauno so stark von diesen Aspekten geprägt ist. Dies hat, wie im Folgenden gezeigt werden soll, darüber hinaus einen philosophischen Hintergrund, der insbesondere für die Charakterisierung der Figur des Vidal zentral ist.

\section{Die Seele als Mühle Und Uhr}

Die konventionelle Interpretation des Zusammenspiels von historischrealistischer und phantastischer Ebene im Film ist eine kompensatorische. Diese Deutung weist der phantastischen Welt des Films einen klar umgrenzten Rahmen zu, innerhalb dessen sie eine ebenso begrenzte Funktion zu erfüllen hat: Ofelia flüchte sich in die phantastische Welt, um die Schrecknisse ihres Alltags zu vergessen. Sie schaffe sich ein phantastisches Universum zur Kompensation ihrer Ängste. Damit würde die Phantastik dem Bereich des Irrealen zugewiesen, sie diente lediglich der Defizitbilanzierung. ${ }^{10}$ Dies generell als die Funktion des Phantastischen zu sehen, ist für Renate Lachmann eine reduktionistische Sicht:

„In neueren Untersuchungen wird die Phantastik als eine Literatur der Kompensation gelesen, die den Zwängen der Aufklärung und der Unterdrückung des aufklärerisch nicht Zulässigen entgegenwirkt, die Beschränkungen aufhebt und das Begehren nach dem Anderen zu artikulieren wagt. Doch greift die Reduktion der Phantastik auf pure Defizitbilanzierung, wie sie für etliche Phantastiktheorien gilt, zu kurz. Denn sie läßt die Ambivalenzen ebenso außer acht wie die ludistische Dimension phantastischer Texte und ihre Tendenz zu semantischer Verschwendung, zu Sinnüberschuß.“ (Lachmann 2002: 25f.)

maten, mir gefiel die Idee, dass ein analoger Mechanismus die Funktionen des Lebens reproduzieren kann. Es gibt wunderbare Geschichten über die europäischen Hersteller von Automaten, Menschen, die kleine Figuren bauten, die gehen und sprechen konnten - oder auch E.T.A. Hoffmanns ,Der Sandmann'. Räderwerke liebte ich als Symbole des Fortschritts." (Arnold 2008: 37)

9 Insbesondere in den beiden Hellboy-Filmen spielen diese Aspekte eine zentrale Rolle: Protagonisten werden immer wieder auf leichte Verspätungen hingewiesen; Rasputin, der Schurke in Hellboy, wird aus einem Labyrinth wiedergeboren, das durch das Blut eines Mannes aktiviert wurde und genauso aussieht wie das des Fauns; Rasputin verfügt über eine Marionette im doppelten Wortsinn, den Maschinen-Nazi Kroenen, der anstelle eines Herzens ein Uhrwerk hat, das aufgezogen und stillgestellt werden kann; die Endkämpfe beider Filme finden in bzw. auf riesigen Uhrwerken statt, deren überdimensionale Räder und Rädchen beide Parteien regelmäßig zu zermahlen drohen.

10 Für diesen Interpretationsansatz siehe z.B. Leweke 2007; Hajduk 2007; Althen 2007. 
Gegen die These der reinen Kompensation eines Defizits spricht in Pans Labyrinth die Tatsache, dass die Off-Stimme, die das einleitende Märchen erzählt, die eines erwachsenen Mannes und nicht des Kindes Ofelia ist. Del Toros Film bleibt dadurch in seiner Darstellung der phantastischen Welt bewusst ambivalent: Mit der zu Anfang von der Off-Stimme erzählten Geschichte wird eine zweite Welt eröffnet, die im Wechsel mit der historischen Ebene dem Film seinen Rhythmus verleiht. ${ }^{11}$ Diese Welt hat auf der Ebene der Bilder dieselbe Existenzberechtigung wie die ,tatsächliche‘. Der OffKommentar rückt sie zwar in zeitliche Ferne, nicht aber in den Bereich der Phantastik: ${ }^{12}$ „Cuentan que hace mucho, mucho tiempo.“ (00:01:27) Der Absolutheitsanspruch der historischen Realität ist durch die Einführung einer Anderswelt gebrochen.

Dennoch erfüllt die phantastische Welt insofern eine kompensatorische Funktion, als es durch sie ermöglicht wird, Erklärungen für ansonsten Unerklärliches zu finden - für den Faschismus. Dafür muss hier der Weg nicht, wie sonst in der Phantastikforschung häufig üblich, über die Psychoanalyse als vielmehr über die Philosophie erfolgen.

Gottfried Wilhelm Leibniz stellt zwei Gleichnisse ins Zentrum seiner Monadenlehre, deren bekanntestes das Mühlengleichnis ist. ${ }^{13}$ Es dient eigentlich der Exemplifizierung seiner Monadologie, der Unterscheidung von beseelten und unbeseelten Vorgängen, kann aber stark vereinfacht als Gegenüberstellung von menschlicher und künstlicher Intelligenz verstanden werden. Die entscheidende Stelle findet sich in $\$ 17$ seiner Monadologie:

„Man muß übrigens zugestehen, daß die Perzeption und was von ihr abhängt durch mechanische Gründe, d.h. durch Figuren und Bewegungen, unerklärbar ist. Angenommen, es gäbe eine Maschine, deren Struktur zu denken, zu fühlen und Perzeptionen zu haben erlaubte, so könnte man sich diese derart proportional vergrößert vor-

11 Der Regisseur selbst nennt in Interviews immer wieder die Bedeutung der Phantastik und von Elementen des Horrorfilms für ihn, unterschlägt aber auch nicht die Möglichkeiten einer kompensatorischen Lektüre: „For me, fairytales have a direct connection to the subconscious." Siehe hierzu z.B. die Interviews unter Festival Cannes.

12 Die Off-Stimme ist auch insofern interessant, als sie im Abspann des Films als „narrador“, also als Stimme eines Erzählers benannt wird. Gleichzeitig ist sie, so wird ebenfalls im Abspann deutlich, auch die Stimme des Fauns, der also, in gewisser Weise, zum Erzähler des Films wird. Festzuhalten ist, dass dieser Film in der Tat über einen Erzähler verfügt, der die Geschichte des Films eröffnet und schließt. Die Kamera nimmt im Film tatsächlich die Rolle eines auktorialen Erzählers ein, ist sie doch stets in der Rolle eines Beobachters. Nur an einer markanten Stelle, auf die weiter unten genauer eingegangen werden soll, nimmt sie die subjektive Perspektive einer Figur ein.

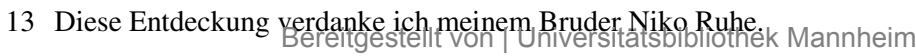


stellen, daß man in sie eintreten könnte wie in eine Mühle. Dies vorausgesetzt, würde man, indem man sie von innen besichtigt, nur Teile finden, die sich gegenseitig stoßen, und niemals etwas, das eine Perzeption erklären könnte.“ (Leibniz 1998: 18f.)

Wahrnehmung und Empfindung sind in einem mechanischen Apparat nicht vorstellbar, obwohl auch in ihm Bewegung stattfinden kann.

Das Zentrum der Mühle, das Vidal bewohnt, lässt sich im Anschluss an diese Überlegungen als überdimensional vergrößertes Symbol für sein Innerstes betrachten: Vidal, der Technokrat, ist nichts weiter als eine Maschine, die zwar in ihren Bewegungen Menschlichkeit vortäuschen kann, aber nichts hat, was ,die Perzeption und was von ihr abhängt“ erklären könnte. Mögen die Monster der phantastischen Welt noch so schrecklich sein, das schlimmste, weil seelenlose und mechanische, Monster ist in diesem Film ein Mensch, dessen Behausung auf sein eigenes Herz verweist, das sich und damit ihn als rein technische Funktion erkennen lässt. ${ }^{14}$ Im Universum del Toros ist Vidal sozusagen die Fortführung von Kroenen, dem Maschinen-Nazi aus Hellboy mit dem Uhrwerk an der Stelle des Herzens. Während im Fall von Kroenen mit seiner Darth-Vader-Maske anstelle eines Gesichts sein maschinelles Wesen in diese geschrieben steht, wahrt Vidal nach außen den menschlichen Schein, bis Mercedes dafür sorgt, dass er sein wahres Gesicht zeigt. Er ist die Personifikation von Faschismus und Totalitarismus.

Sowohl im Zentrum von Leibniz' Mühle als auch bei Vidal wird an der Bewegung ersichtlich, dass Wahrnehmung stattfindet. Dennoch bleibt sie unerklärlich. An dieser Stelle nun setzt die phantastische Ebene des Films an. Ihre Rätsel und Assoziationsspiele (auf die weiter unten eingegangen werden soll), die das Gegenteil der rationalen und faschistischen Welt Vidals sind, bieten Elemente für eine Beantwortung der Fragen nach seiner Motivation. Nicht Ofelia wird damit psychoanalytisch lesbar, vielmehr deutet sie für sich und für den Zuschauer mithilfe ihrer phantastischen Welt, was ansonsten unerklärlich bliebe: ihren Stiefvater und seine Motivation. Sie verfügt, wie die metapoetischen Passagen zu Anfang des Films zeigen, dank der Schulung durch (Märchen-)Literatur über die Fähigkeit zur Wahrnehmung der Verknüpfungsmöglichkeiten von Realität und Fiktion. Sie weist ihr den Weg ins Innere des Menschen.

Leibniz' philosophisches System basiert allerdings noch auf einem weiteren Gleichnis, das im vorliegenden Kontext ebenfalls von hohem Interesse ist, dem so genannten Uhrengleichnis:

14 Vidal ist damit das Gegenteil dessen, was sein Name behauptet - er ist eben nicht „vital“, es sei denn in einem protofaschistischen, Männlichkeit und Kraft verherrlichenden Sinne. 
„Man denke sich zwei Uhren, die fortlaufend ohne die geringste Abweichung übereinstimmen. Die Übereinstimmung kann auf dreierlei Arten herbeigeführt sein: Entweder die beiden Werke sind durch eine technische Vorrichtung so miteinander verbunden, dass das eine vom andern mechanisch abhängig ist und daher nicht von ihm abweichen kann. Oder es ist ein beaufsichtigender Mechaniker vorhanden, der beide fortlaufend reguliert. Oder, drittens, die beiden Uhren sind mit solcher Kunstfertigkeit und Präzision gemacht, dass eine Abweichung ausgeschlossen ist.“ (Störig 1993: 339f.)

Leibniz erklärt in diesem Gleichnis das Zusammenwirken von Leib und Seele. Er selbst glaubte an die dritte Variante; die Occasionalisten sahen Gott in der Rolle des Mechanikers (vgl. Mittelstraß 2004: 1067f.).

Vidals sorgfältige Reinigung und Wartung seiner Taschenuhr ist das Werk des ,beaufsichtigende[n] Mechaniker[s]“, denn damit sorgt er dafür, dass sein Körper und seine mechanische Seele fortlaufend übereinstimmen. ${ }^{15}$ Er setzt sich in die Rolle Gottes, der nach Leibniz für die Synchronisierung von Körper und Seele Sorge trägt. In Vidals Welt ist davon nur noch die Sorge um ein Uhrwerk geblieben, das im Takt des Faschismus tickt. Als ihr ,oberster Uhrmacher“ maßt er sich die Kontrolle über die Welt an. ${ }^{16}$

15 Damit fällt Vidal hinter Leibniz zurück und macht sich sozusagen den pervertierten Standpunkt der Occasionalisten zu eigen. Andererseits liegt darin, worauf auch Hubertus Busche hinweist, eine durchaus ,klassische“ Fehllektüre von Leibniz: „Daß Leibniz mit seiner Präetablierten Harmonie eine derartige Vermittlungsposition einnimmt, wird selten erkannt, denn traditionell wird dieses berühmte Lehrstück in dem Sinne verstanden, daß Gott, als der gleichsam perfekte Uhrmacher der Welt, Leib und Seele wie zwei Uhren von vorneherein synchronisiert habe, ohne daß es hierfür einen natürlichen Grund gebe. Nach diesem Klischee bestünde die Leibnizsche Erklärung allein in der dogmatischen Behauptung, daß der göttliche Wundermann, weil er ja alles kann, auch dieses übernatürliche Kunststück per Dekret, also durch seine absolute Macht vollbracht habe. Ausgerechnet Leibniz, der den Okkasionalisten Wunderglauben vorwarf, hätte selbst die psychophysische Harmonie zu erklären beabsichtigt durch ein unerklärliches göttliches Wunder!“ (Busche 2009: 66)

16 Guillermo del Toro ist, so machen die Interviews mit ihm deutlich, ein ausgesprochen belesener Regisseur, der seinen Schauspielern zum besseren Verständnis ihrer Rollen z.B. literarische Texte empfiehlt. Die philosophische Vertiefung erscheint daher in diesem Kontext durchaus naheliegend. 


\section{5 (Wieder-)Geburt IM Geiste Der Geschichte}

Ofelias Entdeckung der phantastischen Welt erfolgt unmittelbar nach ihrer Ankunft in der Mühle, in der Vidal sich mit seinen Truppen niedergelassen hat und in der sie nun mit ihrer Mutter und dem kleinen Bruder leben soll, dessen Geburt insbesondere Vidal ungeduldig erwartet.

In der ersten Nacht in der Mühle folgt Ofelia der Stabheuschrecken-Fee wie vor ihr Lewis Carrolls Alice dem weißen Kaninchen. ${ }^{17}$ Statt im Kaninchenbau landet sie aber im titelgebenden Labyrinth. Hier trifft Ofelia erstmals auf den Faun, dessen steinernes Abbild sie zuvor vervollständigt hatte. Er meint, in ihr sofort die Prinzessin Moana zu erkennen.

Del Toros Anderswelt folgt den Regeln des Märchens. Entsprechend erlegt der Faun Ofelia drei Prüfungen auf, mittels derer sich erweisen soll, dass sie tatsächlich die verschollene Prinzessin der Unterwelt ist. Zur Übermittlung der Aufgaben erhält Ofelia ein großes Buch, dessen Seiten jedoch leer sind. Sie werden sich, so der Faun, für sie allein sichtbar mit der Beschreibung ihrer Aufgaben füllen. Der Faun bezeichnet das Buch als „El libro de las encrucijadas“, als „das Buch von den Scheidewegen“. Dieser Name kann, wie zu zeigen sein wird, tatsächlich als Zusammenfassung aller Aufgaben Ofelias dienen.

Die Prüfungen, die das Mädchen in der Anderswelt zu bestehen hat, sind stets in komplexer Weise mit Ereignissen in der realen Welt des Films verflochten, in denen allerdings nicht Ofelia, sondern ihr erwachsenes Alter Ego Mercedes, die Helferin der Partisanen, im Zentrum steht. Die Montage führt die auf diese Beziehung verweisenden Szenen dabei nicht parallel, sondern stellt sie stets leicht zeitversetzt nebeneinander.

Ofelias erste Aufgabe ist es, eine riesige Kröte, die unter den Wurzeln eines Baumes lebt und ihn zum Absterben bringt, mittels dreier vergifteter Bernsteine zu überwältigen und ihr einen goldenen Schlüssel abzunehmen. Ofelia meistert diese Aufgabe erfolgreich. Allerdings muss sie dafür in einen niedrigen, engen und schlammigen Gang unter die Wurzeln des Baumes kriechen, wo die Kröte haust. ${ }^{18}$ Das neue, grüne Kleid und die neuen Schuhe, die ihre Mutter ihr kurz zuvor stolz überreicht hatte, sind eine wenig geeignete Bekleidung für diese Aufgabe. Ofelia zieht das Kleid daher aus und hängt es in die Äste des Baumes, so als wollte sie dem toten Baum bereits damit etwas von seinem natürlichen Grün zurückgeben. ${ }^{19}$

17 In der Tat wird insbesondere im Internet immer wieder auf den Einfluss von Lewis Carrolls Alice's Adventures in Wonderland (1865) und Through the LookingGlass, and What Alice Found There (1871) auf den Film del Toros verwiesen, ohne dass dieser Spur aber weiter nachgegangen würde.

18 Der Weg unter die Wurzeln des Baumes zur Kröte ähnelt auffällig Alices Weg in den Kaninchenbau und damit ins Wunderland.

19 Es ist auffallend, dass die Protagonistin den ganzen Film hindurch stets grüne

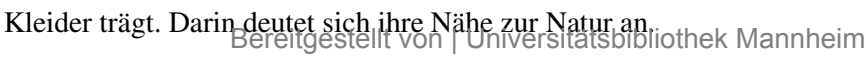


Das Kleid ist aber noch unter einem anderen Gesichtspunkt interessant: Es ist als augenzwinkernder Hinweis darauf zu verstehen, dass Ofelia tatsächlich in mancherlei Hinsicht eine Enkelin von Lewis Carrolls Alice ist. Alice trägt in der international berühmten Zeichentrickversion dieses Klassikers (1951) ein auffallend ähnliches Kleid (Abb. 2 und 3).

\section{Abbildung 2: Ofelia}

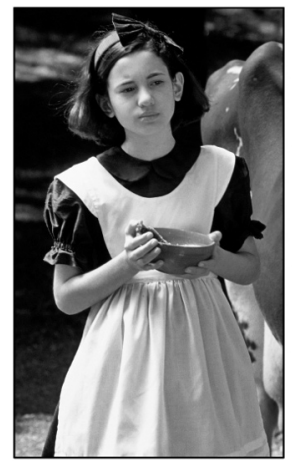

Quelle: (C) Senator Film
Abbildung 3: Alice

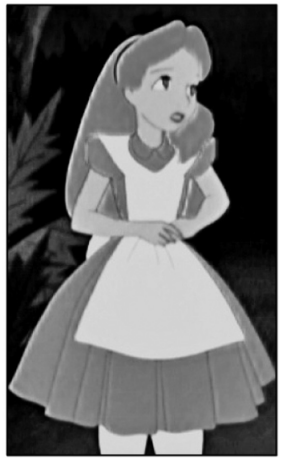

Quelle: (C) Disney

Verleih GmbH 2007

Der Baum, in dessen Innerstes Ofelia bei ihrer Aufgabe vordringt (Abb. 4), wird doppelt markiert: Der wie durch Zauberhand im „Libro de las encrucijadas" erschienene Text bezeichnet ihn als den Baum, unter dem sich zu Anbeginn der Zeit Menschen und Zauberwesen zum Schlaf versammelten und der damit von einer Zeit kündet, als Realität und Phantastik miteinander noch nicht im Widerspruch standen, so wie der Film dies erneut in Szene setzt.

Vor allem weckt der Baum aber eine andere Assoziation: die eines arból genealógico, eines Stammbaums. Der mächtige Baum, der nur zwei Hauptäste ausgebildet hat, steht für Spanien und den das Land bestimmenden Konflikt zwischen den dos Españas, den „zwei Spanien“. Dieser im 19. Jahrhundert entstandene Konflikt zwischen - stark vereinfacht gesagt - konservativen und liberalen Kräften, mündet schließlich in den Bürgerkrieg, an dessen Ende die Handlung des Films angesiedelt ist. Dieser Baum, so wird durch Ofelias Aufgabe deutlich, ist an der Wurzel vergiftet und stirbt daher. Ofelias erfolgreiche Erfüllung ihrer Aufgabe ermöglicht ihm nun ein neues, von der Vergiftung befreites Leben. ${ }^{20}$

20 Der sterbende Baumriese weckt optisch noch eine weitere Assoziation: Seine beiden Hauptäste sind so gebogen, dass sie sich wie die Hörner des Fauns ausnehmen. 


\section{Abbildung 4: Der Baumriese, unter dessen}

Wurzeln die Kröte haust

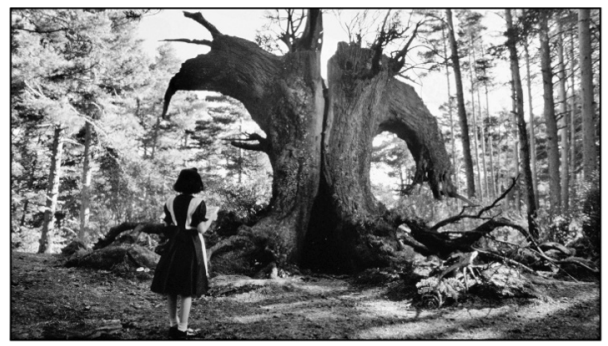

Quelle: (C) Senator Film Verleih GmbH 2007

Ofelias Wiederauftauchen aus dem engen Gang unter den Wurzeln des Baumes nach ihrer gehorsamen und genauen Erfüllung der ersten Aufgabe könnte als ihre zweite Geburt interpretiert werden. Wie nach ihrer tatsächlichen Geburt geht Ofelia schmutzig aus dem neuerlichen Geburtskanal hervor und wird - wie nach einer Entbindung üblich - sogleich gebadet. Ofelia ist aber nicht nur selbst neugeboren, sie wird auch zur Geburtshelferin eines neuen, vom alten Gift befreiten Spanien. In der Ermöglichung einer anderen Geschichte für ihr vom Bruderzwist geplagtes Land besteht der eigentliche, tiefere Sinn ihrer Aufgaben.

Der Schlüssel, den sie der Kröte entreißt, korrespondiert mit der Parallelhandlung um Mercedes. Diese gelangt unterdessen als Vidals Dienstbotin in den Besitz des einzigen Schlüssels zum Vorratsraum, in den Vidal die gesamten Essensvorräte des Dorfes hat bringen lassen, um so zu verhindern, dass von dort weitere Nahrungslieferungen an die Partisanen erfolgen. Mercedes fertigt von diesem Schlüssel ein Doppel an, das sie an ihren Bruder, den Führer der Partisanen, übergibt.

\section{Im Zentrum DER ZeIT}

In derselben Nacht - Ofelia ist ohne Essen ins Bett geschickt worden - besteht ihre zweite Aufgabe darin, an die Wand ihres Zimmers mithilfe eines Stücks Kreide, ${ }^{21}$ das sie vom Faun erhielt, eine Tür zu zeichnen, die sich dann für sie öffnet und den Weg in die Anderswelt freigibt. Ihre Zeit dort wird von einer Sanduhr gemessen; ist sie abgelaufen, schließt sich die Tür und es gibt keinen Rückweg mehr für Ofelia. Durch die Tür gelangt sie in einen unterirdischen Raum, in dem ein Festmahl aufgetischt ist. Am Kopf des Tisches thront eine unheimliche, augenlose Gestalt, die del Toro in sei-

21 In einem Film, der eine begeisterte Leserin und die Kraft der Phantasie ins Zentrum rückt, ist es durchaus konsequent, dass die Kreide, also ein Schreibwerkzeug, die Türen in die Anderswelt öffnet. 
nen Skizzen stets als den Pale Man bezeichnet (Abb. 5). Der Pale Man hat seine Augäpfel auf einem Teller vor sich liegen. Der Faun hat Ofelia zuvor eingeschärft, dass sie die Speisen nicht anrühren dürfe und dass das, was sie in dem unterirdischen Raum erwarte, „no [...] humano“ (00:50:57) sei. Diese Szene, zu der ebenfalls eine Parallelhandlung abläuft, ist nicht nur die Mitte des Films, sondern auch sein Zentrum.

Auf der realistischen Ebene des Films findet zwischen beiden Aufgaben ein Bankett in der Mühle statt, das Vidal für die Honoratioren des Dorfes gibt. Die Einladung soll dazu dienen, ihnen die Rationierung der Nahrungsmittel für die Dorfbewohner bekanntzugeben. Bei diesem realistischen Mahl nimmt Vidal denselben Ehrenplatz ein wie der Pale Man in der Unterwelt. Mercedes sorgt währenddessen gemeinsam mit den anderen Dienstboten für das Wohl der Gäste und lauscht aufmerksam Vidals Ausführungen, um sie später den Partisanen überbringen zu können.

\section{Abbildung 5: Ofelias Begegnung mit dem}

Pale Man

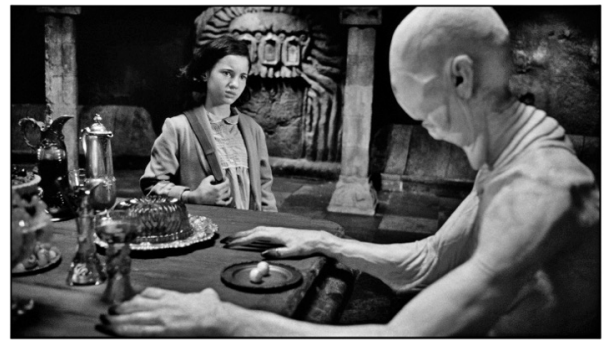

Quelle: @ Senator Film Verleih GmbH 2007

Ofelia bestaunt zunächst das unterirdische Festmahl und die Ausstattung des Raumes. Dabei fallen ihr an der Wand Fresken im mittelalterlichen Stil auf, die den Pale Man als kinderfressendes Monster zeigen. Darüber hinaus entdeckt sie einen Berg von Schuhen, der in der Filmhandlung nicht weiter motiviert ist. Er lässt sich deuten als Hinweis auf die Fotos von den Bergen von Schuhen der ermordeten KZ-Häftlinge. ${ }^{22}$ Der Pale Man und seine Untaten werden damit in einen noch unmenschlicheren faschistischen Kontext gestellt als der, dem auch Hauptmann Vidal angehört.

Ofelia gelingt es, mithilfe des von der Kröte erbeuteten Schlüssels eine Art Schließfach zu öffnen, das wiederum eine ins Monströse verzerrte Darstellung des Fauns ist: Die drei „Schließfächer“ sind wie die drei aufgerissenen Münder des steinernen Fauns, dessen vier Augen Ofelia anstarren. Die Feen, die Ofelia begleiten, weisen ihr das mittlere Schließfach, sie entscheidet sich aber für das linke, in dem sie einen Dolch findet, den sie einsteckt.

22 In diesen Bildern ist ein Hinweis auf Alain Resnais' berühmten dokumentarischen Film Nuit et brouillard (1955) zu sehen. 
Hierin liegt ihr erster Verstoß gegen die Regeln des Fauns, der ihr die Feen mit den Worten „Dejaos guidar por ellas“ (00:50:45) übergeben hatte. Auf dem Rückweg zögert sie vor der reich gedeckten Tafel und isst schließlich drei Trauben, trotz des expliziten Verbots des Fauns. Sofort erwacht der Pale Man zum Leben, setzt die Augäpfel in die dafür vorgesehenen Stellen in seinen Handflächen ein und macht sich an ihre Verfolgung. Er verschlingt dabei zwei der Feen. Ofelia und der letzten verbleibenden Fee gelingt es mit letzter Kraft, ihm zu entkommen.

Auf bildlicher Ebene zeigt sich bereits zu Anfang des Films, dass die Statuen des Fauns mit Vidal korrespondieren (Abb. 6 und 7). Damit wird die Statue mit den Schließfächern in gewisser Weise zu einer weiteren Darstellung Vidals. Der Dolch, in dem sich Mercedes' Messer spiegelt, mit dem sie Vidal später verletzen wird, kommt auf der Bildebene gleichsam aus seinem eigenen Mund. Auf der realistischen Ebene des Films entspricht diese Sequenz Vidals Entdeckung von Mercedes' Verrat. Er will sie foltern, damit sie ihre Komplizen verrät. Mercedes verfügt jedoch über ein Messer, das sie stets im Umschlag ihrer Schürze verborgen hält - das Äquivalent zu dem Dolch, den Ofelia dem Schließfach entnimmt - und es gelingt ihr so nicht nur, sich zu befreien, sondern auch Vidal zu verletzen. Sie stößt ihm das Messer in den Mund und damit zurück an den Ort, aus dem Ofelia den Dolch holt und aus dem zu Anfang die Fee schlüpft, und reißt es gewaltsam wieder heraus. Dadurch fügt sie Vidal eine Wunde zu, die seinen Mundwinkel ins Groteske verlängert. Er wird damit in offensichtlicher Weise zu dem Monster, das er immer gewesen ist. ${ }^{23}$

23 Das ganze Ausmaß seiner Unmenschlichkeit wird in einer weiteren Szene offenbar: Zurück in seinem Raum im Inneren der Mühle näht er diese Wunde selbst mit einem grob aussehenden Faden und einer Nadel. Trotz der Schmerzen, die er offenkundig verspürt, kommt er dieser Aufgabe ohne zu zögern nach. Die entstehende Naht versteckt er unter einem Pflaster, dennoch ist für den Zuschauer deutlich geworden, dass Vidal zwar aus Fleisch und Blut sein mag, dabei aber von Fäden zusammengehalten wird, die eines Frankensteins würdig wären. Im Gegensatz zu Mary Shelleys Wissenschaftler, der die namenlose Kreatur zusammensetzt, ist es hier allerdings Vidal, also das Monster selbst, das sich verarztet

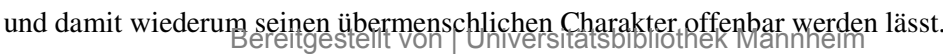


Abbildung 6: Ofelia und der Faun

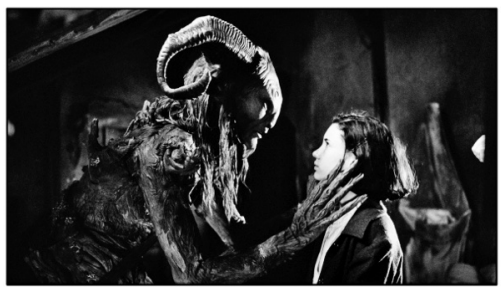

Quelle: (c) Senator Film Verleih GmbH 2007
Abbildung 7: Ofelia und Vidal

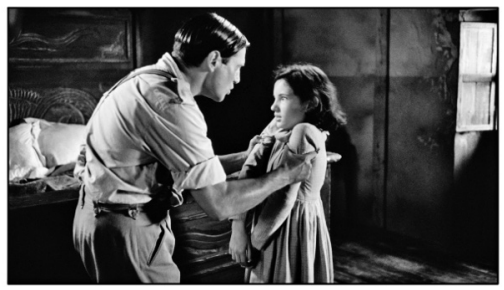

Quelle: (C) Senator Film Verleih GmbH 2007

Der Pale Man, Vidals Alter Ego in der Unterwelt, wird durch die unterschiedlichen Interpretationsansätze, die sich für ihn anbieten, zu einer sehr komplexen Figur. Einerseits spiegelt er durch seine Position am Tisch und vor dem lodernden Kaminfeuer Vidal. Andererseits stand für seine Darstellung ein Gemälde aus Francisco de Goyas Pinturas negras (1819-1823) Pate (Abb. 8). Es stellt eine wichtige Referenz zur kulturellen Tradition Spaniens dar (vgl. Kermode 2006).

Abbildung 8: Goya, Saturno devorando a sus hijos

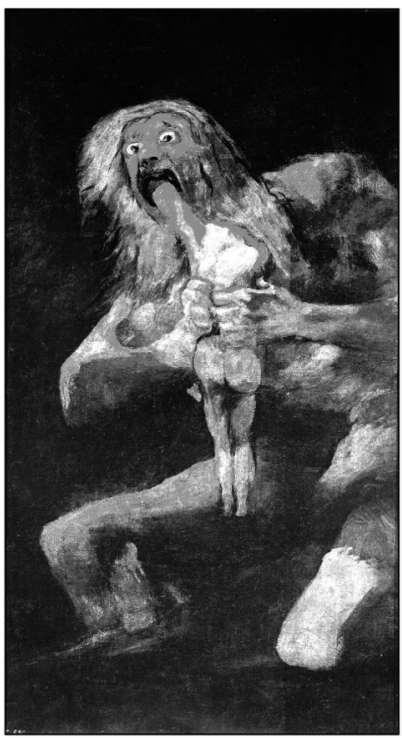

Quelle: Internet

Dieser Bezug ist in doppelter Hinsicht interessant: Einerseits spiegelt die Darstellung Saturns, der seinem Kind gerade den Kopf abgebissen hat, exakt die Art und Weise, in der der Pale Man die Feen zerfleischt. Andererseits 
verweist die Tatsache, dass es sich hier um eine mythologische Szene handelt, auf einen tiefer liegenden Kontext.

Saturn ist in der römischen Mythologie durch den Mord an seinem eigenen Vater an die Macht gekommen. Ihm wird seinerseits geweissagt, er werde durch die Hand eines seiner Kinder sterben. Daher verschlingt er vorsorglich seinen Nachwuchs, bis auf Jupiter, den seine Frau vor ihm verbergen kann und der ihn schließlich ermorden wird. Das Äquivalent zu Saturn in der griechischen Mythologie ist Kronos, der in der Volksetymologie bereits früh mit Chronos, dem Gott der Zeit, gleichgesetzt wurde. Pan ist, so eine der Erklärungen seiner Herkunft, ein Sohn des Zeus und damit ein Enkel des Kronos. ${ }^{24}$

Dieser Verweis führt zurück zum Anfang meiner Ausführungen: Der erste Spielfilm Guillermo del Toros heißt La invención de Cronos; in ihm spielen Fragen des Alterns und der ablaufenden Lebenszeit eine gewichtige Rolle. In all seinen Filmen zeigt sich del Toros Faszination für Uhrwerke und Mechanik, stets ist die Zeit von herausragender Bedeutung.

Im Zentrum dieses Films nun sitzt mit dem Pale $\mathrm{Man}^{25}$ als Personifikation von Saturn/Kronos die Inkarnation der Zeit selbst. ${ }^{26}$ Er spiegelt Vidal, den von Uhren und der Zeit besessenen Protagonisten des Films, dessen Innerstes, wie bereits gezeigt worden ist, gleichsam aus einem Uhrwerk be-

24 Im Film ist die mythologische Ebene für den aufmerksamen Zuschauer an einer einzigen Stelle angedeutet: Als Ofelia zum ersten Mal in das Labyrinth hinabsteigt, ruft sie in die Stille „Echo“, woraufhin der Faun erscheint. In der griechischen Mythologie wird von der Nymphe Echo berichtet, dass Pan in sie verliebt gewesen sei, sie sich ihm jedoch verweigert habe.

25 Die an religiöse Fresken erinnernden Bilder im Raum des Pale Man, die ihn als Kinderfresser zeigen, gewinnen in diesem Kontext eine zusätzliche Bedeutung, handelt es sich doch offensichtlich um die Bilder von den Morden an seinen eigenen Nachkommen.

26 In Lewis Carrolls Alice im Wunderland spielt die Zeit ebenfalls eine zentrale Rolle: Das weiße Kaninchen ist von seinem ersten Auftreten an sehr besorgt darüber, dass es sich verspäten könnte. Die zentrale Szene bei Carroll ist die Teeparty beim verrückten Hutmacher und dem Märzhasen. Der verrückte Hutmacher erläutert, dass er sich mit der (personifizierten) Zeit gestritten und sie daher die Zeiten durcheinander gebracht habe, sodass nun ständig teatime sei. Auch hier gibt es also interessante Korrespondenzen.

Mit Lewis Carroll zitiert del Toro einen Autor, der bei Weitem nicht nur in seinen literarischen Texten an der Zeit interessiert war, sondern auch über wissenschaftliche Expertise bezüglich der Zeit verfügte: „His interest in time as a physical concept, rather than a metaphysical one, drew on his scientific expertise. $\mathrm{He}$ participated in the Victorian effort to fix an International Date Line, a venture launched by the Royal Society of London. Carroll's paper on the subject was published in the Macmillan journal Nature, still the principal journal for key new 
steht und der gleichzeitig ein Rädchen im größeren Getriebe des faschistischen Spaniens ist. ${ }^{27}$ Es könnte daher kein besseres Symbol für ihn geben als Kronos, den Herrn über die Uhren. ${ }^{28}$

Vidal wird am Ende des Films tatsächlich seine Stieftochter Ofelia töten. Auch Kronos verschlingt vermeintlich all seine Kinder, bis auf Zeus, den seine Gattin vor ihm versteckt, indem sie ihm anstelle des Kindes einen in Windeln gewickelten Stein gibt, den er verspeist. Er wird später von seinem Sohn getötet, den er meint, verschlungen zu haben. Vidal wird ebenfalls von Ofelias erwachsenem Alter Ego, von Mercedes und den anderen Partisanen, ermordet werden, die dies stellvertretend für Ofelia tun und die Vidal glaubte, vernichtet zu haben.

Die Figur des Saturn/Kronos spielt nicht nur in der Mythologie, sondern auch in ihrer Verwendung durch die Psychoanalyse eine wichtige Rolle. Bei C.G. Jung figuriert Saturn als einer der Archetypen, der für einen übermächtigen, verschlingenden Vater steht. In diesem Kontext gehen Analytiker wie John W. Crandall von einem Kronos-Komplex aus, den Crandall als „destructive ingestive process which hinders the [child's] capacity to exist separately and autonomously“ (Crandall 1984: 110) definiert. Maria Martinez-Ortiz, die dieser Frage für den vorliegenden Film nachgegangen ist, kommt zu dem Schluss, Vidal sei ,,a well-rounded metaphor of the ,Cronus complex““ (Martinez-Ortiz 2010). Ob man die Existenz dieses Komplexes nun anerkennt oder nicht, der Film legt eine psychoanalytische Interpretation Vidals im Sinne eines ausgeprägten Vaterkomplexes jedenfalls sehr nahe. Diese Deutung der Figur wird allerdings erst durch das Zusammenspiel der historisch-realistischen und der phantastischen Ebene möglich.

Der Film entwickelt sich von seinem Anfang wie von seinem Ende her konsequent auf die zentrale Szene des Pale Man hin bzw. aus ihr heraus. Ofelias Tod an beiden „Enden“ des Films und die Tatsache, dass ihr Blut in der ersten Szene rückwärts fließt, verweisen darauf, dass die Zeit hier, ausgehend von Kronos, dem Herrn über sie, rückwärts bzw. vorwärts verläuft. Die beiden Hälften des Films verhalten sich in mancher Hinsicht spiegelbildlich zueinander; so korrespondieren etwa die jeweils drei Auftritte des

27 Diesen Vorwurf des blinden Gehorsams macht Vidal der Arzt: „Es que obedecer para obedecer, así, sin pensarlo, eso solo lo hacen gentes como usted, Capitán.“ (01:20:43)

28 Mit Blick auf den mythologischen Hintergrund lässt sich auch Vidals Unwilligkeit, über seinen Vater zu sprechen, deuten: Wenn er, und sei es auch nur auf symbolischer Ebene, seinen Vater getötet hat, dann muss ihm ein Gespräch über ihn notwendigerweise unangenehm sein. Zugleich ist seine Aussage „Nunca tuve un reloj“ (00:40:58) durchaus in diesem Zusammenhang lesbar - wenn er der Herrscher über die Zeit ist, so kann nicht bereits sein Vater über ein Instrument zu ihrer Messung verfügt haben. Die Uhr allerdings, die Vidal besitzt und eifrig pflegt, hat einen auffallenden Sprung im Glas und könnte daher durchaus identisch sein mit der, die sein Vater angeblich zerschmettert hat. 
Fauns miteinander, aber auch das Steinauge zu Anfang entspricht Vidals brechendem Auge am Schluss des Films. Die Tatsache, dass Ofelia Vidal bei ihrer ersten Begegnung die falsche Hand reicht, wofür er sie tadelt, könnte ein Indiz dafür sein, dass die Welt hier sozusagen spiegelverkehrt ist. ${ }^{29}$ In der falschen, linken Hand wird zugleich augenzwinkernd die politische Positionierung der Protagonisten angedeutet.

\section{Wahrnehmung und Phantastik}

Wie bereits zu Anfang erwähnt, geht es in El laberinto del fauno zentral um die Wahrnehmung. In der zitierten Passage von Leibniz, dem so genannten Mühlengleichnis, geht es ebenfalls um Perzeption. Eine Maschine ist, so Leibniz, dazu nicht imstande, sie kann bloß funktionieren. Diese Beobachtung lässt sich auf die Figur des Hauptmanns Vidal übertragen: Er ist im Stande, innerhalb eines vorgegebenen Systems zu funktionieren, seine Einhaltung zu kontrollieren und damit als das zu agieren, was bereits sein Vater war, ,un gran militar“ (00:40:39). In der Leibniz'schen Logik agieren hier „,nur Teile [...], die sich gegenseitig stoßen, und niemals etwas, das eine Perzeption erklären könnte“ (Leibniz 1998: 19). Die Grenzen des Systems sind damit für Vidal die Grenzen seines Bewusstseins und seines Verständnisses. Gehorsam ist für ihn das oberste Gebot, und einen Verstoß dagegen ist er unfähig zu verstehen.

Seine Wahrnehmung ist dabei aber noch auf einer anderen Ebene gestört, wie die Parallelsetzung mit dem Pale Man in eindrucksvoller Weise vor Augen führt: Vidals monströser Doppelgänger kann seine Augen, das zentrale Wahrnehmungs- und Kontrollorgan, herausnehmen und sich damit jeglicher Wahrnehmung verweigern, solange in den Grenzen eines Systems

29 In dieser „Spiegelverkehrtheit“ der Welt könnte wiederum ein weiterer Bezug zu Lewis Carroll liegen, in diesem Fall zu Through the Looking Glass: Bereits in Alice in Wonderland hatte Alice stets zu ihrer eigenen Überraschung spontan Lieder und Gedichte mit ,falschen“ Texten deklamiert. Erst Alices Weg hinter den Wohnzimmerspiegel führt sie dann aber in ein Reich, in dem die Zeit selbst ebenso wie die Erinnerung in beide Richtungen verlaufen kann: „,Living backwards!' Alice repeated in great astonishment. ,I never heard of such a thing!" ,- but there's one great advantage in it, that one's memory works both ways. ' ,I'm sure mine only works one way. ' Alice remarked. ,I can't remember things before they happen. "It's a poor sort of memory that only works backwards, ' the Queen remarked. ,What sort of things do you remember best?' Alice ventured to ask. ,Oh, things that happened the week after next,' the Queen replied in a careless tone. ,For instance, now,' she went on, sticking a large piece of plaster on her finger as she spoke, ,there's the King's Messenger. He's in prison now, being punished: and the trial doesn't even begin till next Wednesday: and of course the crime comes last of all “ “ (Carroll 2005: 100f.) 
gehandelt wird. Er wird damit gleichsam zum Symbol für den blinden Gehorsam. Befolgt Ofelia getreulich die Regeln des Fauns, der sich hier, ebenso wie Vidal, verdoppelt, bleibt der Pale Man statisch. Er sieht sie nicht, denn das Hinschauen ist überflüssig, solange sie die an sie gestellten Erwartungen erfüllt. ${ }^{30}$

Als Ofelia sich zur Regelübertretung entschließt und die Trauben vom Tisch des Pale Man isst, erwacht dieser zum Leben. Ihre Handlung ist dabei durchaus ambivalent: Einerseits kann man es als klassische Illustration dessen sehen, dass selbst Ofelia durch den Hunger, den sie verspürt, korrumpiert zu werden und den Verführungen, die von Vidal und dem durch ihn repräsentierten System ausgehen, zu verfallen droht. Andererseits liegt darin eine bewusste Übertretung der Regeln des Fauns, eine Auflehnung gegen den absoluten Gehorsam, der ihr abverlangt wird. Dieser Verstoß wird ganz konsequent damit bestraft, dass zwei der Feen, die für die überbordende Kraft der Phantasie stehen, verschlungen werden und somit Ofelias Universum der Märchen, gegen das auch Vidal und ihre Mutter opponieren, geschwächt wird. ${ }^{31}$

Der Pale Man ist nach seinem Erwachen nicht sofort handlungsfähig, vielmehr muss er erst seine Augäpfel in die dafür vorgesehenen Höhlen in seinen Handflächen einsetzen. Sodann hält er sich die Hände mit den Flächen nach außen an die Stellen, an denen eigentlich die Augen sitzen (Abb. 9) und nähert sich mit drohenden, aber deutlich zögernden Schritten Ofelia.

Das Bild des Auges in der Hand(-fläche) ist ein in vielen Kulturen verwendetes Symbol. In ihm kombinieren sich die beiden wichtigsten Wahrnehmungs- und Erkenntnisinstrumente des Menschen, sodass es gemeinhin als Bild für das Streben nach Allwissenheit und Allmacht, für Kontrolle gesehen wird. ${ }^{32}$

30 In diesem Punkt spiegeln sich erneut Ofelia und Mercedes bzw. Vidal und der Pale Man: Für Vidal ist Mercedes „no [...] más que una mujer“, daher war sie für ihn, wie sie selbst sagt, stets unsichtbar. Erst ihr Verrat lässt sie für ihn sichtbar werden.

31 Eine bereits zitierte Szene, in der die Fee gemeinsam mit dem Faun etwas isst, das wie rohes Fleisch aussieht und vor dem Ofelia sich ekelt, suggeriert jedoch, dass die Fee möglicherweise mehr ist als nur die Inkarnation der Phantasie - sie ist eine Spionin, die der Faun Ofelia mitgegeben hat, um ihren Gehorsam zu prüfen. In der Tat fliegt sie, kaum dass Ofelia sich gegen das von ihr gezeigte Schließfach entschlossen hat, sofort zum Pale Man, um ihn auf diese Verfehlung aufmerksam zu machen. Eine Reaktion bleibt jedoch aus. Die phantastische Welt selbst ist demnach bereits affiziert von der Bosheit der realistisch-historischen Ebene. Ihre zumindest für die romanischen Länder zentrale Exponentin - die den cuentos de hadas titelgebende Fee - hat sich korrumpieren lassen.

32 In den islamischen Kulturen ist die so genannte Hand der Fatima ein Schutztalisman, der vor dem bösen Blick bewahren soll. In den meso-amerikanischen 
Abbildung 9: Der Pale Man ist zum Angriff

bereit

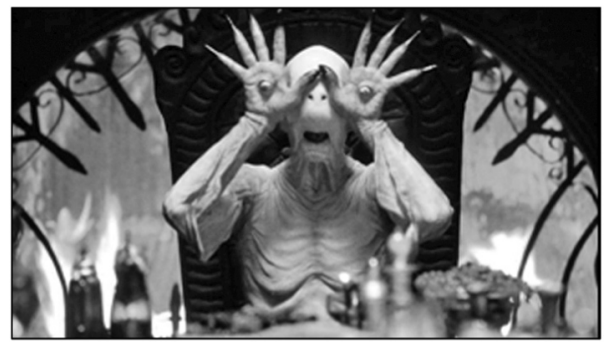

Quelle: @ Senator Film Verleih GmbH 2007

Um die Allmacht und Allwissenheit des Pale Man ist es allerdings, so zeigt der Film deutlich, gerade aufgrund der Lokalisierung seiner Augen, schlecht bestellt: Handelt er (im wahrsten Sinne des Wortes), so kann er nicht sehen, sieht er, so kann er nicht handeln. Wahrnehmung und Handlung schließen sich gegenseitig aus. Zugleich nimmt der Pale Man und mit ihm Vidal die Welt nicht über den dafür eigentlich vorgesehenen Sinn - die Augen - zur Kenntnis, sondern über die Hände, die die Welt im doppelten Wortsinn manipulieren und instrumentalisieren. Er nimmt Menschen wie Mercedes, aber auch den Arzt oder den Stotterer stets erst im Moment ihrer Regelverletzung wahr, sozusagen beim Verstoß gegen seine Manipulationen. Seine Beschäftigung mit ihnen erfolgt dann auf der reinen Handlungsebene, indem er sie foltert oder ermordet. In diesen Momenten ist er wiederum zur Wahrnehmung nur in unzureichender Weise fähig: Er tötet zwei Bauern, ohne ihren Fall vorher genauer in Augenschein zu nehmen, wofür er anschließend seine Soldaten verantwortlich macht; er tötet den Arzt, der der Einzige ist, der seine Frau und Ofelias Mutter retten könnte und verurteilt damit seine eigene Frau zum Tode.

In der Tat ist Vidal in der Logik des Films in ganz fundamentaler Wiese zur Wahrnehmung unfähig: Die zweite Ebene des Films, das Reich der Phantastik, in dem er sich in der Figur des Fauns und des Pale Man vervielfacht hat, kann er nicht sehen. Dieser Aspekt wird in einer wiederum ambivalenten Szene des Films offensichtlich. Während Ofelias letztem Gespräch mit dem Faun, in dem sie sich nun vollends gegen dessen Prüfungen und Regeln auflehnt, indem sie sich weigert, ihren Bruder zu opfern, wird sie von Vidal beobachtet. Die Kamera nimmt seine Perspektive ein und sieht nun nur noch Ofelia, die vor einem unsichtbaren Gegenüber, mit dem sie spricht, zurückweicht. Betrachtet man diese Szene mit einer Haltung, die in der Phantastik reine Kompensation und Defizitbilanzierung sieht, so muss man davon ausgehen, dass sich darin der irreale Charakter der phantasti-

Kulturen, vor allem bei den Azteken, existiert ebenfalls ein solches Symbol, das dem culto de la muerte zugeordnet wird. 
schen Welt in objektiver Weise entbirgt. Die Parallelwelt kann tatsächlich nur von Ofelia gesehen werden. Es ist aber gerade keine objektive Perspektive, die die Kamera hier einnimmt, sondern vielmehr die subjektive Vidals. Er repräsentiert in diesem Film die Person mit der eingeschränktesten, problematischsten Wahrnehmung, ja in ihm ist, wenn man die Leibniz'sche Idee konsequent denkt, nichts, „das eine Perzeption erklären könnte“ (Leibniz 1998: 19). Noch einmal wird die Einseitigkeit dieses Charakters ausgestellt. $\mathrm{Zu}$ einer Beobachtung, die über die Grenzen des für ihn Vorstellbaren hinausgeht, ist er nicht in der Lage.

\section{Verletzte Regeln}

Der Sinn von Ofelias Aufgaben besteht wie im Märchen vermeintlich in der unbedingten Befolgung der ihr auferlegten Regeln und damit im Erlernen von blindem Gehorsam. Hier kreuzt sich ihre Lektüreerfahrung in der Welt der Märchen mit der Realität, verlangt der verabscheute Stiefvater ihr doch dasselbe ab wie die Märchen ihren Protagonisten. Das Buch mit ihren Aufgaben, das der Faun ihr überreicht, wird damit ganz buchstäblich zu einem „Buch der Scheidewege“. Ofelia entscheidet sich bereits nach der ersten Aufgabe dafür, die Regeln zu durchbrechen, und bringt so deren Erfüllung und nicht zuletzt sich selbst in akute Gefahr.

Ofelias letzte Aufgabe soll darin bestehen, so will es der Faun, ihm ihren wenige Tage alten Bruder zu bringen und mithilfe seines Blutes - „solo un poco de sangre" (01:40:53) - das Labyrinth zu öffnen und Ofelia damit den endgültigen Zugang zur Anderswelt zu verschaffen. Auf allegorischer Ebene steht der Bruder für das Neue Spanien, das nach dem Willen Vidals ein faschistisches, kontrolliertes und kontrollierbares Land werden soll und dessen Geburtswehen der Zuschauer auf der historischen Ebene des Films miterlebt. In einem solchen patriarchalischen System ist für Carmen kein Platz mehr; sie zu opfern wird billigend in Kauf genommen, wie es Vidal selbst dem Arzt gegenüber bereits geäußert hatte. Wenn man so will, könnte in dem Schicksal, das ihrem Kind zugedacht ist, und in seiner Überhöhung zum Neuen Spanien auch der Grund für Carmens massive Übelkeit liegen. Sie, die keine überzeugte Faschistin zu sein scheint, wehrt sich innerlich gegen diese Instrumentalisierung ihres Kindes. ${ }^{33}$ Nicht nur die Geburt des Bruders ist blutig und für die Mutter - die damit für das Alte Spanien steht tödlich, ihr gehen bereits starke Blutungen voraus, die das Leben von Mutter und Kind in Gefahr bringen. In diesen Elementen lässt sich die allegorische Umsetzung des Bürgerkriegs zwischen Nationalspaniern und Republikanern sehen, dessen Blutzoll hoch ist und der nur über den Versuch der Aus-

33 Das Unbehagen der schwangeren Frau gegenüber dem Kind, das sie erwartet, findet sich als wiederum phantastisch aufgelöstes Motiv auch in Roman Polanskis Rosemary's Baby, auf den dieser Aspekt zu, verweisen, scheint, 
löschung einer Partei zum Ziel gelangen kann. Ofelia verhindert allerdings die weitere Vereinnahmung des Bruders durch Vidal, d.h. das faschistische Regime, und überantwortet ihn gleichsam postum an die Partisanen, damit sie ihn, wenngleich Sohn eines Faschisten und im Geiste des Faschismus geboren, zum Sinnbild für das andere, das Neue Spanien in ihrem und in Ofelias Sinne machen können.

Ofelia weigert sich auch dem Pan gegenüber, ihren Bruder zu opfern. Er, der das letzte ist, was von ihrer Ursprungsfamilie geblieben ist, sie aber auch für immer an Vidal bindet, soll bei ihr bleiben. Für ihn, so betont der Faun, gibt sie ihr geheiligtes Recht auf die Herrschaft der Unterwelt auf, obwohl er der Grund dafür sei, dass sie „humillada e ignorada“ (01:41:26) worden sei. In der Verteidigung ihres Bruders steht Ofelia nolens volens auch für die Interessen ihres verhassten Stiefvaters ein. Vidal wird es ihr nicht danken, sondern sie stattdessen erschießen. Die untrennbare und ambivalente Verbindung beider Spanien wird in der Figur des Bruders, der mehr eine Projektionsfläche als eine tatsächliche Figur ist, deutlich: Das republikanische und das faschistische Spanien mögen sich zwar ideologisch voneinander getrennt haben und einander bis aufs Blut bekämpfen, sie sind aber zugleich durch unauflösbare familiäre Bande aneinander gebunden.

Für die sterbende Ofelia öffnet sich dadurch, dass ihr Blut und nicht das ihres Bruders in das Labyrinth tropft, ein Tor zur Anderswelt. Sie betritt einen prunkvollen Raum, in dem auf turmhohen Thronen ihre Mutter und ihr Vater sitzen. Ihr Vater bestätigt ihr, dass sie die Prüfung, die gerade im Schutz ihres Bruders bestand, erfolgreich absolviert habe. Ihr Blut habe sie für seines vergossen. Die Prüfung hatte also paradoxerweise - und ganz in der Logik vieler Märchen - die Auflehnung gegen die Regeln des Systems zum Ziel.

Naheliegend ist aber auch eine andere Interpretation: Durch ihren Opfertod öffnet sich das Labyrinth; der Sieg der Partisanen und ein Wiedersehen mit ihren Eltern werden ermöglicht, somit ein in ihrem Geiste - dem der Auflehnung gegen das totalitäre System - positives Ende. Wäre hingegen das Blut ihres Bruders vergossen worden, hätte sich ganz im Sinne Vidals eine faschistische Welt des blinden Gehorsams geöffnet.

Der Film schließt den von ihm gezeichneten Kreis: Ofelias Tod und die um sie weinende Mercedes am Rande des Labyrinths sind das letzte Bild, bevor die Off-Stimme vom Anfang des Films wieder einsetzt, um von Ofelias langer und glücklicher Herrschaft im Reich ihres Vaters zu berichten. Ihr Aufenthalt in der Realität hat Spuren hinterlassen - so wird betont, während die Kamera auf den Baumriesen schwenkt, unter dessen Wurzeln Ofelia ihre erste Prüfung absolviert hat. An einem seiner toten Äste öffnet sich nun, gegen alle Wahrscheinlichkeit, eine Blüte. Diese und andere Erinnerungen an Ofelia sind allerdings ,,visible solo por aquel que sepa donde mirar" (01:47:28), sind also nur für denjenigen sichtbar, der zur Wahrnehmung fähig ist. 


\section{LITERATUR}

Althen, Michael (2007): „Das blutige Werk von Nadel und Faden“, in: Frankfurter Allgemeine Zeitung 47 vom 24.02.2007, S. 35.

Arnold, Frank (2008): „Interview. Mein Hellboy ist autobiografisch“, in: epd Film 10/2008, S. 37.

Busche, Hubertus (2009): „Einführung“, in: Gottfried Wilhelm Leibniz, Monadologie, Berlin: Akademie, S. 49-80.

Carroll, Lewis (2005): Alice: Through the Looking-Glass and What She Found There, London: Walker.

Crandall, John W. (1984): „The Cronus-Complex“, in: Clinical Social Work Journal 12.2, S. 108-117.

Festival Cannes, http://www.festival-cannes.fr/fr/mediaPlayer/8694.html vom 22.02.2010.

Filmjournal, http://filmjournal.net/clydefro/-2007/01/12/pans-labyrinth vom 22.02.2010.

Fuchs, Cynthia: „A Symbol of Transition: Interview with Guillermo del Toro", in: http://www.popmatters.com/pm/feature/a-symbol-of-tran sition-interview-with-guillermo-del-toro vom 30.03.2010.

Hajduk, Thomas (2007): „Amnesie im Wunderland. Pans Labyrinth“, in: filmrezension.de - Online-Magazin für Filmkritik vom 16.03.2007, http://filmrezension.de/+frame.shtml?/filme/pans_labyrinth.shtml

Jones, Jo Elwyn/Gladstone, J. Francis (1998): The Alice Companion: A Guide to Lewis Carroll's Alice Books, New York: New York UP.

Kermode, Mark (2006): „Pain should not be sought - but it should never be avoided“, in: The Observer vom 05.11.2006, http://www.guardian.co.uk/ film/2006/nov/05/features.review1

Lachmann, Renate (2002): Erzählte Phantastik. Zu Phantasiegeschichte und Semantik phantastischer Texte, Frankfurt a.M.: Suhrkamp.

Leibniz, Gottfried Wilhelm (1998): Monadologie (hg. von Hartmut Hecht), Stuttgart: Reclam.

Leweke, Anke (2007): „Die hohe Kunst der Verdrängung. Guillermo del Toros erschütternder Film Pans Labyrinth", in: Die Zeit vom 01.03.2007, http://www.zeit.de/2007/10/Fantasy-Filme

Martinez-Ortiz, Maria (2010): „Fantasy and Myth in Pan's Labyrinth: Analysis of Guillermo del Toro's Symbolic Imagery“, in: First Global Conference on Magic and the Supernatural, http://www.inter-disci plinary.net/wp-content/uploads/2010/02/martinezpaper.pdf vom 30.03. 2010.

Mittelstraß, Jürgen (2004): „Okkasionalismus“, in: Jürgen Mittelstraß (Hg.), Enzyklopädie Philosophie und Wissenschaftstheorie, Bd. 2, Stuttgart: Metzler, S. 1067-1068.

Störig, Hans Joachim (1993): Kleine Weltgeschichte der Philosophie, Frankfurt a.M.: Fischer.

$U G O$, http://www.ugo.com/ugo/html/article/?id=16348 vom 22.02.2010. 
Wikipedia, http://en.wikipedia.org/wiki/Pan's_Labyrinth vom 22.02.2010.

\section{FILME}

Blade II (2002) (USA, R: Guillermo del Toro)

El espinazo del diablo (2001) (E/MEX, R: Guillermo del Toro)

El laberinto del fauno (2006) (E/MEX, R: Guillermo del Toro)

Hellboy (2004) (USA, R: Guillermo del Toro)

Hellboy - The Golden Army (2008) (USA, R: Guillermo del Toro)

La invención de Cronos (1993) (MEX, R: Guillermo del Toro)

Mimic (1997) (USA, R: Guillermo del Toro)

Nuit et brouillard (1955) (F, R: Alain Resnais) 
Bereitgestellt von | Universitätsbibliothek Mannheim Angemeldet Heruntergeladen am | 26.10.17 09:51 